undifferentiated panniculitis and with the disease duration of 1 week to 13 years In addition to general clinical study, serum concentrations of a-1 antitrypsin, amylase, lipase, ferritin, creatinephosphokinase were determined, computed tomography of the chest organs, immunological, ultrasound scanning of the skin and SCF of the node area, tuberculosis tests and pathomorphological study of skin biopsy from the node area were performed.

Results: : as a result of laboratory and instrumental examination, the following diagnoses were made: EN associated with infection (72 people - group 1), lipodermatosclerosis (LDS) (40 -group 2), idiopathic lobular panniculitis (ILP) (32 - group 3), Löfgren's syndrome (SL) (49-group 4). During the follow-up period, 6 deaths occurred (3.1\%): in a patient with LDS due to acute heart failure and in 5 patients with ILP due to the activity of the disease which led to the development of cardiopulmonary pathology and disseminated intravascular coagulation (DIC) syndrome. In group 1, during the observation period, UE recurred in 18 people $(25 \%)$, the probable causes were: hypothermia (6), exacerbation of chronic tonsillitis (6), acute respiratory viral infections (ARVI) (4), stress (1), a rapid decrease in the dose of glucocorticoids (GC) (1), the cause is unknown (2). In group 2, recurrence occurred in 19 patients $(47.5 \%)$ due to the lack of anti-inflammatory therapy (plaquenil) (10), trauma (4), stress (3), and weight lifting (2). There was no recurrence in 7 patients in this group, however, nodes would not disappear completely, which is obviously due to the absence of plaquenil. In group 3, recurrence was registered in 24 cases $(75 \%)$ and it was associated with insufficient effect of the ongoing anti-inflammatory therapy (9), a decrease in blood glucose to minimal doses (6), hypothermia (6) and the absence/cancellation of anti-inflammatory therapy (3). In group 4, recurrence of nodes was registered in 14 cases (28.5\%), possible causes: cancellation of GC (9) and cooling/ARVI(5). Conclusion: in the observed group of patients with panniculitis mortality was $3.1 \%$. The main causes of recurrence in EN were viral-bacterial infections, and in case of ILP, LDS and SL it was insufficient effect/absence of anti-inflammatory therapy. Disclosure of Interests: None declared

DOI: 10.1136/annrheumdis-2020-eular.1433

\section{AB1023 1 CARDIAC MRI IN HYPERFERRITINAEMIC DISEASE STATES REVEALS MYOCARDIAL INFLAMMATION NOT IDENTIFIED BY ECHOCARDIOGRAPHY}

S. El-Ghazali ${ }^{1}$, H. Wilson-Morkeh ${ }^{1}$, A. Porter ${ }^{1}$, S. Thapa ${ }^{1}$, J. Mason ${ }^{1,2}$ M. Fontana ${ }^{3}$, A. Singh ${ }^{4}$, G. Cole ${ }^{2}$, T. Youngstein ${ }^{1,2}$. ${ }^{1}$ Hammersmith Hospital, Rheumatology, London, United Kingdom; ${ }^{2}$ National Heart and Lung Institute, Imperial College London, London, United Kingdom; ${ }^{3}$ Royal Free Hospital, National Amyloidosis Centre, London, United Kingdom; ${ }^{4}$ Royal Free Hospital, Rheumatology, London, United Kingdom

Background: Acutely unwell adult patients with hyperinflammatory hyperferritinaemic states are typically challenging to diagnose. Case series suggest that cardiac involvement may be common (up to $20 \%$ ) but the phenotype has not been well characterised ${ }^{1}$

The elevation of cardiac biomarkers suggests cardiac involvement, but are non-specific in acute illness. Cardiac MRI (CMR) offers the ability to characterise the myocardium and identify inflammation, and modern motion-corrected sequences now allow the assessment of patients who may struggle to breathhold in the recovery from acute illness.

Objectives: We report 3 patients who underwent CMR in the acute phase of illness with raised cardiac biomarkers.

Methods: Case records of acutely ill patients with hyperferritinaemia from two major London centres were reviewed and cases who had undergone CMR in the acute phase of illness were identified.

Results: 3 cases were identified from a cohort of 22, we report CMR findings from differing aetiologies of hyperferritinaemic states:

Case 1: A female in her 60s presented acutely unwell with fever, swollen joints and salmon pink rash. Ferritin was raised at 50574ug/L (20-300ug/L), troponin I 384ng/L (<34ng/L) and Brain Natriuretic Peptide (BNP) 324ng/L (<159ng/L). Echocardiography was normal. However CMR with T2 mapping revealed several small areas of raised signal consistent with myocardial inflammation. A diagnosis of systemic Adult Onset Stills Disease (AOSD) was made. She received IV methylprednisolone and anakinra with normalisation of cardiac biomarkers.

Case 2: A male in his 20s with known SLE with associated end stage renal failure requiring transplant. He had a previous prolonged admission secondary to $\mathrm{HLH}$. He presented with chest pain and concave shaped ST elevation on ECG. Troponin peak 2168ng/L, BNP 1334ng/L. Peak ferritin 1300ug/l.

He was initiated on colchicine for likely pericarditis. Echocardiography showed a dilated left ventricle and mildly increased wall thickness, but overall systolic function within normal limits.

CMR reported nodular patchy late gadolinium enhancement in the mid inferoseptum and inferior wall associated with areas of raised T2 mapping values. NM cardiac rest gated PET reported abnormal FDG uptake to the myocardium with sites including the apical inferior wall, apical RV insertion point and basal septal/ anterior right ventricular walls. Features were deemed in keeping with active myocarditis.

He responded to colchicine with improved troponin, and was discharged with close follow up.

Case 3: A male in his 20s presented with septic shock attributed to meningococcal septicaemia requiring ITU admission. Troponin was elevated at $>9000 \mathrm{ng} / \mathrm{L}$ Bloods demonstrated raised ferritin and features consistent with HLH were identified.

CMR reported elevated native myocardial T1/T2 signal of the lateral and mid-anterior walls in keeping with myocardial oedema. Pericardium adjacent to the anterolateral wall had elevated T1/T2 signal with hyperenhancement on delayed enhancement imaging. Tissue characterisation was in keeping with an acute myopericarditis process.

In addition to broad spectrum antibiotics to treat his underlying infection, he received therapy for $\mathrm{HLH}$ including methylprednisolone, anakinra and IVIG. He subsequently made a good recovery to treatment.

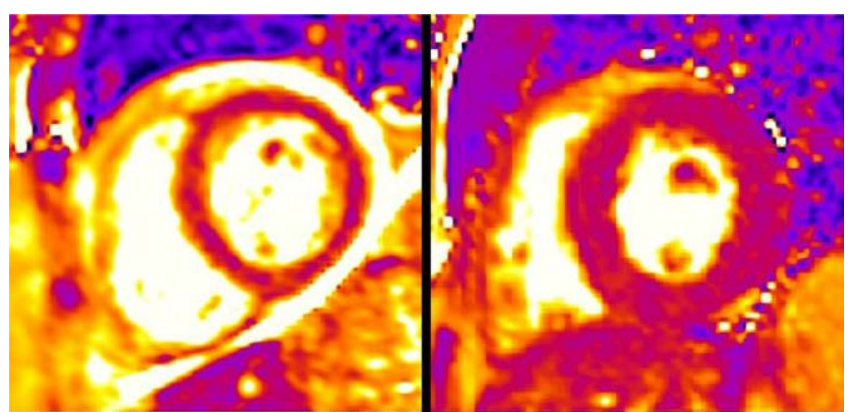

Left panel shows a short-axis $\mathrm{T} 2$ map from Case 1. There is heterogeneity to $\mathrm{T} 2$ mapping values with multiple areas $>55 \mathrm{~ms}$ consistent with myocardial oedema. There is also a pericardial effusion. Right panel shows a short-axis T2 map from Case 2. There is again heterogeneity to T2 mapping with multiple areas $>55 \mathrm{~ms}$ consistent with myocardial oedema. There is also LVH consistent with hypertension.

Conclusion: CMR in acute illness with hyperferritinaemia reveals abnormal tissue characterisation with myocardial inflammation, even when echocardiography is normal. We suggest CMR may be a useful test to expand our understanding of hyperferritinaemic disease states.

\section{References:}

[1] M Gerfaud-Valentin et al. Myocarditis in Adult-Onset Still Disease. Medicine (Baltimore) 2014 Oct; 93(17): 280-289

Disclosure of Interests: None declared

DOI: 10.1136/annrheumdis-2020-eular.2666

\section{$\mathrm{AB} 1024$ \\ EVALUATION OF CLINICAL FEATURES IN PATIENTS DIAGNOSED WITH JUVENILE AND ADULT-ONSET FAMILIAL MEDITERRANEAN FEVER}

D. Erdem Gürsoy ${ }^{1}$, H. H. Gezer², S. Acer Kasman², N. Öz², A. Ozer², M. T. Duruöz ${ }^{2}{ }^{1}$ Adıyaman Training and Research Hospital, Rheumatology, Adıyaman, Turkey; ${ }^{2}$ Marmara University School of Medicine, Department of Physical Medicine and Rehabilitation, Rheumatology Division, Istanbul, Turkey

Background: Familial Mediterranean Fever (FMF), which is more common in groups in the Mediterranean basin, is a monogenic auto inflammatory disease characterized by recurrent attacks of febrile peritonitis, pleuritis and arthritis. Objectives: The aim of this study is to investigate the clinical features of patients diagnosed with juvenile and adult-onset Familial Mediterranean Fever (FMF). Methods: Patients with FMF were included in the study consecutively without sample selection. Data about age, sex, disease duration (month), symptom duration, age at diagnosis, diagnosis delay time, comorbid diseases, and medications were noted. Patients with onset of symptoms $\leq 20$ years old were classified as juvenile-onset, those $>20$ years old were classified as adult-onset FMF. The frequency and characteristics of attacks and the presence of amyloidosis will be recorded

Disease activity was assessed with the PRAS disease activity score. The Health Assessment Questionnaire (HAQ) and SF-36 were used to evaluate physical disability and quality of life, respectively.

The descriptive analysis was done for all parameters. Differences between categorical variables were assessed by Chi-square test and Fisher's exact test. The Mann Whitney-U test was used to compare two sample means. $\mathrm{P}<0.05$ accepted as significant. SPSS 20.0 (Statistical package for social sciences for Windows 20.0) program was used for the statistical analysis.

Results: The mean age of 86 patients ( 63 female, 23 male) with FMF was 38.38 (SD: 12.13 ) years. The patients with the juvenile-onset FMF were $26.7 \%$ of the patients. 
There were no differences between juvenile and adult-onset FMF groups in terms of gender, frequency of attacks, duration of attacks, acute phase values between attacks, colchicine dose, presence of colchicine resistance, and presence of amyloidosis $(p>0.05)$. The latency to diagnosis was significantly higher in patients with adult-onset FMF $(p<0.005)$.

The PRAS disease activity scores were significantly higher in the juvenile-onset FMF group ( $p=0.001)$. There were no significant differences between the two groups in terms of SF-36 and HAQ scores ( $p>0.05$ ).

Conclusion: While there were no differences between juvenile and adult-onset FMF patients in terms of quality of life and functional disability, the PRAS disease activity scores were higher in patients with juvenile-onset FMF.

References:

[1] Shinawi M, Brik R, Kepten I, Berant M, Gersoni B. Familial Mediterranean fever: high gene frequency and heterogenous disease among Israeli-Moslem Arab population. J Rheumatol 2000;27:1492-5.

Disclosure of Interests: None declared

DOI: 10.1136/annrheumdis-2020-eular.6106

\section{AB1025 EVALUATION OF ANXIETY AND DEPRESSION LEVELS IN PATIENTS WITH FAMILIAL MEDITERREAN FEVER}

\section{S. Ergulu Eșmen ${ }^{1} .{ }^{1}$ Konya Education and Research Hospital, Rheumatology, Konya, Turkey}

Background: Anxiety and depression are common problems in chronic rheumatological diseases (1). Familial Mediterranean Fever (FMF) is characterized by recurrent fever and serosal inflammation attacks.

Objectives: The aim of the study was to evaluate the levels of anxiety and depression in patients diagnosed with FMF, and to examine its relationship with drug compliance and attack frequency.

Methods: Sixty female patients aged 18 years or older who were consecutively admitted to the rheumatology outpatient clinic and diagnosed as FMF according to the Tel-Hashomer Classification criteria were included. Patients' age, gender, body mass index (BMI), educational status, disease duration, drugs used and frequency of attacks were recorded. Beck anxiety scale and Beck depression scale were used to determine the level of anxiety and depression.

Results: Sixty female patients with a mean age of $32.03 \pm 10.03$ and an mean disease duration of $9 \pm 6.9$ years were included in the study. The patients were divided into minimal, mild, moderate and severe according to their anxiety level. According to the level of anxiety, minimal anxiety was observed in $18(30 \%)$, mild $9(15 \%)$, moderate $12(20 \%)$ and severe anxiety in $10(16.7 \%)$ patients. Data related to anxiety are given in table 1 . The patients were divided into minimal, mild, moderate and severe depending on the level of depression. Depending on the level of depression, minimal depression was seen in 34 (56.7\%), mild 13 $(21.7 \%)$, moderate $8(13.3 \%)$ and severe depression in $4(6.7 \%)$ patients. Data related to depression are given in table 2 .

Table 1. Data about anxiety

\begin{tabular}{lccccc}
\hline & Minimal & Mild & Moderate & Severe & $P$ \\
\hline Age (years) (mean \pm SD) & $30.82 \pm 10.7$ & $26.22 \pm 8.5$ & $34.42 \pm 10.2$ & $33.4 \pm 9.7$ & 0.28 \\
BMI $\left(\mathrm{kg} / \mathrm{m}^{2}\right)($ mean $\pm \mathrm{SD})$ & $25.53 \pm 5.05$ & $19.88 \pm 2.7$ & $25.03 \pm 4.91$ & $26.03 \pm 3.4$ & 0.02 \\
Disease duration (years) & $11.93 \pm 9.6$ & $8.67 \pm 4.0$ & $10.5 \pm 6.7$ & $4,89 \pm 3,7$ & 0.13 \\
Marriatal status (Married) & $13(70)$ & $2(22.2)$ & $10(83.3)$ & $9(90)$ & 0,06 \\
Child status (\%)(having child) & $12(70.6)$ & $1(11.1)$ & $8(72.2)$ & & 0,006 \\
Time of the last attack (\%) & $2(11.8)$ & $2(22.2)$ & $4(33.3)$ & $4(50)$ & 0,72 \\
$\quad 2$ weeks ago & $4(23.5)$ & $1(11.1)$ & $2(16.7$ & - & \\
3 months ago & $7(41.2)$ & $4(44.4)$ & $4(33.3)$ & $3(37.5)$ & \\
$>6$ months & & & & &
\end{tabular}

Tablo 2. Data about depression

\begin{tabular}{lccccc}
\hline & Minimal & Mild & Moderate & Severe & P \\
\hline Age (years) (mean \pm SD) & $32.79 \pm 9.89$ & $28.83 \pm 8.93$ & $32.63 \pm 10.5$ & $36.5 \pm 14.3$ & 0.61 \\
BMI $\left(\mathrm{kg} / \mathrm{m}^{2}\right.$ ) (mean $\left.\pm \mathrm{SD}\right)$ & $25.68 \pm 5.09$ & $22.06 \pm 3.2$ & $23.81 \pm 4.33$ & $26.35 \pm 2.8$ & 0.076 \\
Disease duration (years) & $10.52 \pm 8.06$ & $8.5 \pm 4.6$ & $8.67 \pm 4.6$ & $1.5 \pm 1$ & $<0.00$ \\
Marriatal status (Married) & $25(73.5)$ & $8(61.5)$ & $7(87.5)$ & $3(75)$ & 0,63 \\
Child status (\%)(having child) & $23(71.9)$ & $5(38.5)$ & $7(87.5)$ & $3(75)$ & 0,08 \\
Time of the last attack (\%) & & & & & 0,94 \\
$\quad 2$ weeks ago & $6(18.2)$ & $2(16.7)$ & $2(28.6)$ & $2(50)$ & \\
3 months ago & $8(24.2)$ & $1(8.3)$ & $1(14.3)$ & $1(25)$ & \\
$>6$ months & $12(36.4)$ & $5(41.7)$ & $2(28.6)$ & $1(25)$ & \\
& & & & & \\
\hline
\end{tabular}

Conclusion: In the case of a chronic disease such as FMF, which has a younger patient population than other rheumatic diseases, anxiety and depression should be evaluated during routine outpatient clinic administration and psychiatric support should be provided if necessary.

\section{References:}

[1] Deger SM, Ozturk MA, Demirag MD, et al. Health-related quality of life and its associations with mood condition in Familial Mediterranean Feve patients. Rheumatol Int. 2011;31(5):623-8.

Disclosure of Interests: None declared

DOI: 10.1136/annrheumdis-2020-eular.2680

\section{AB1026 \\ INFLAMMATORY MANIFESTATIONS IN PATIENTS WITH HUMAN LEUKOCYTE ANTIGEN-B*51 POSITIVE AND WITHOUT BEHÇET'S DISEASE}

M. Duarte ${ }^{1}$, J. Sousa Morais ${ }^{2}$, R. Faria ${ }^{1,3}$, B. Guerra Leal ${ }^{3,4}$, A. Marinho ${ }^{1,3}$,

J. Correia ${ }^{1,3}$, F. Farinha $^{1}$, P. Pinho Costa ${ }^{3,4,5}$, B. Martins Da Silva ${ }^{3,4}$

C. Vasconcelos ${ }^{1,3} \cdot{ }^{1}$ Centro Hospitalar e Universitário do Porto, Unidade de Imunologia Clínica, Porto, Portugal; ${ }^{2}$ Hospital de São Marcos, Braga, Serviço de Medicina, Braga, Portugal; ${ }^{3}$ Abel Salazar Biomedical Sciences Institute - University of Porto, UMIB, Porto, Portugal; ${ }^{4}$ ICBAS, Laboratório de Imunogenética, Porto, Portugal; ${ }^{5}$ INSA, Porto, Portugal

Background: Human leukocyte antigen (HLA) $B^{\star} 51$ allele is the most important genetic factor in susceptibility to Behçet's disease (BD), an immune-mediated systemic disorder of unknown etiology, characterized by recurrent episodes of inflammatory manifestations. In fact there is a considerable clinical overlap of BD with autoinflammatory syndromes. As it's known, the majority of HLA-B*51 positive individuals do not develop BD. But do these individuals also present inflammatory manifestations?

Objectives: Characterize the group of individuals in our hospital with positive HLA-B*51, without BD diagnosis and review whether inflammatory manifestations are present in these individuals.

Methods: A retrospective study of HLA-B ${ }^{\star} 51$ positive patients between 2000 and 2019. Genomic DNA was obtained from peripheral blood and HLA genotyping was performed using a PCR with Sequence Specific Primers (PCR-SSP) methodology. From the group of $289 B^{\star} 51$ positive patients, BD diagnosis were excluded. Demographic and clinical data were collected by review of clinical files in December 2019.

Results: 176 patients, mean age of $48.5 \pm 16.5$ years (5 to 84 years). Most were female $(68 \%)$. The HLA study was motivated by multiple diagnostic suspicions: spondyloarthritis (SpA, 25.0\%), BD (22.7\%) and systemic sclerosis (SSc $10.8 \%)$. The mean time elapsed since the immunogenetic study was 8.3 years with 12 deaths recorded. 69 (39.2\%) subjects had no diagnosis for immune-mediated disease (IMD). Of the other 107 patients, the majority had 1 IMD (64.5\%), and the rest were diagnosed with 2 to 4 IMD. The most frequent IMD were SpA $(20.8 \%)$, psoriasis $(10.4 \%)$, psoriatic arthritis $(9.7 \%)$, SSc $(9.7 \%)$ and rheumatoid arthritis (7.1\%). Autoantibodies were detected in 94 individuals (53.4\%): antinuclear antibodies (64 patients), rheumatoid factor (26 patients) and CCP antibodies (11 patients). In 55 individuals no inflammatory manifestation was identified but the $68.8 \%$ of them presented between 1 to 7 manifestations:

\begin{tabular}{|c|c|c|c|}
\hline \multirow{2}{*}{$\begin{array}{l}\text { System involved } \\
\text { Ears, nose and throat }\end{array}$} & \multirow{2}{*}{$\begin{array}{l}\text { Clinical manifestation } \\
\text { Chronic rhinitis }\end{array}$} & \multicolumn{2}{|c|}{ Frequency } \\
\hline & & $32(13.2 \%)$ & $\Sigma=76(31.4 \%)$ \\
\hline & Recurrent tonsillitis & $20(8.3 \%)$ & \\
\hline & Other & $24(9.9 \%)$ & \\
\hline \multirow{3}{*}{$\begin{array}{l}\text { Cutaneous, mucous and } \\
\text { serous }\end{array}$} & Recurrent oral aphtous ulcers & $34(14.0 \%)$ & $\Sigma=57(23.6 \%)$ \\
\hline & Serositis & $10(4.1 \%)$ & \\
\hline & Other & $13(5.4 \%)$ & \\
\hline \multirow[t]{2}{*}{ Neurological } & Chronic headache & $34(14.0 \%)$ & $\Sigma=36(14.9 \%)$ \\
\hline & Asseptic meningitis & $2(0.8 \%)$ & \\
\hline Ocular & Ocular inflammation & $31(12.8 \%)$ & $\Sigma=31(12.8 \%)$ \\
\hline Urinary & Recurrent cystitis & $15(6.2 \%)$ & $\Sigma=15(6.2 \%)$ \\
\hline \multirow[t]{3}{*}{ Vascular } & Venous thromboembolism & $7(2.9 \%)$ & $\Sigma=14(5.8 \%)$ \\
\hline & Aneurysm & $6(2.5 \%)$ & \\
\hline & Spontaneous coronary dissection & $1(0.4 \%)$ & \\
\hline \multirow[t]{2}{*}{ Rheumatic } & Arthromyalgia & $6(2.5 \%)$ & $\Sigma=9(3.7 \%)$ \\
\hline & Gout & $3(1.2 \%)$ & \\
\hline Constitutional & Recurrent fever syndrome & $1(0.4 \%)$ & $\Sigma=1(0.4 \%)$ \\
\hline Digestive & Recurrent abdominal pain & $1(0.4 \%)$ & $\Sigma=1(0.4 \%)$ \\
\hline Lymphatic & Axillary and inguinal adenopathies & $1(0.4 \%)$ & $\Sigma=1(0.4 \%)$ \\
\hline Pulmonary & Chronic pulmonary infiltrate & $1(0.4 \%)$ & $\Sigma=1(0.4 \%)$ \\
\hline
\end{tabular}

Conclusion: Inflammatory manifestations are common in HLA-B*51 positive individuals, even in those without BD diagnosis. Further research is needed, considering other HLA alleles associated with increased risk of BD and including control groups.

References:

[1] Burillo-Sanz S, Montes-Cano M, García-Lozano J, et al. Behçet's disease and genetic interactions between HLA-B ${ }^{\star} 51$ and variants in genes of autoinflammatory syndromes. Sci Rep 2019;9:2777.

[2] McGonagle D, McDermott MF. A Proposed Classification on the Immunolog ical Diseases. PLoS Med 2006;3(8):e297. 